NOTA CIENTIFICA

\title{
MEJORAMIENTO DE LOMBRICES NATIVAS POR CRUZAMIENTO CON EISENIA FOETIDA
}

Francisco Sales Dávila *

\section{RESUMEN}

El presente trabajo se efectuó en el marco del Proyecto Sistema Integral de Producción Agraria que está realizando el Centro Regional de Investigación del IIAP en Ucayali (CRI-IIAP-Ucayali), ubicado en la carretera Federico Basadre km 12400 Pucallpa, durante el periodo de abril a setiembre de 1995.

Los objetivos del estudio consistieron en tratar de incrementar el tamaño de lombrices mediante el cruzamiento sexual para incrementar el peso y la producción de humus.

La evaluación para el cumplimiento de los objetivos se programó en seis fases: cuarentena, siembra de lombrices, incubación, crecimiento, reproducción de híbridos (F1) y prueba final con la progenie (F2).

El estudio se desarrolló hasta la segunda fase, concluyéndose que no se logró el cruzamiento en el tratamiento T3 (lombriz Eisenia foetida por lombriz nativa).

Sin embargo, en los tratamientos T2 (Eisenia foetida por lombriz Eisenia foetída) y el tratamiento T1(nativa por nativa) se logró la cópula, postura de cápsula, eclosión y crecimiento (pequeños y juveniles), en lombrices del mismo género y especie.

* Investigador del Centro Regional de Investigaciones de Ucayali. 


\begin{abstract}
The present work was done within the frame of the Integral System Project of Agrarian Production which is being carried out by the Centro Regional de Investigación of IIAP-Ucayali, on F. Basadre highway at the $12400 \mathrm{~km}$ in Pucallpa, April to September,1995.
\end{abstract}

The objectives of this study consisted on trying to increase the size of earthworms through sexual intercourse in order to increase the weight and the production of humus.

The evaluation for the fullfillment of the objectives was programmed in six phases: quarantine, breeding of earthworms, incubation, growth, hybrid reproduction (F1) and a final test with the offspring (F2).

This study was carried out up to the second phase, concluding by not crossbreeding the Eisenia foetida earthworm with the native earthworrn in treatment 3.

Neverthless, in treatment T2 (Eisenia foetida for the Eisenia foetida earthworm) and treatment $\mathrm{T} 1$ (native for native) the mating the encapsulating posture, the eclosion and the growth of both small and young in earthworms of the same genre and species.

\title{
1. INTRODUCCION
}

En el mundo existen más de 8000 especies de lombrices, sin embargo son pocas las que pueden vivir en cautiverio, la lombriz Eisenia joetida es una de las especies que se adapta a estas condiciones, es muy prolífera y voraz pero es pequeña en longitud y peso, lo que es una desventaja productiva en comparación con las lombrices nativas amazónicas; motivo por el cual se hace necesario tratar de lograr el cruzamiento a fin de obtener una especie mejorada en voracidad y tamaño que produzca mayor cantidad de humus con fines de abono y carne para el balanceo de alimentos para animales menores. 


\section{REVISION DE LITERATURA}

Según Grasse (1975), la clasificación es la siguiente:

$\begin{array}{lll}\text { Reino } & : & \text { Animal. } \\ \text { Sub-reino } & : & \text { Metazoa,(metazoo). } \\ \text { Clase } & : & \text { Oligochaeta,(Oligoquetos). } \\ \text { Orden } & : & \text { Opistóporos. } \\ \text { Series } & : & \text { Lombricinos. } \\ \text { Género } & : & \text { Eisenia. } \\ \text { Especie } & : & \text { Eisenia,foétida. } \\ \text { Nombre común } & : & \text { Lombriz de estiércol o lombriz cebra. }\end{array}$

Para el mismo autor, los oligoquetos comprenden más de 1800 especies, seis de ellas son comunes en gran parte del mundo, esto se debe a la costumbre humana de transportar plantas de un lugar a otro.

Meinicke (1989).- La describe como un animal que está provisto de todas las condiciones para sobrevivir en un ambiente, está equipada con todos los atributos físicos para cumplir su trabajo con una fría eficiencia y desprendimiento de cualquier exceso de bulto. Se puede notar que Eisenia foetida es fría y viscosa debido a que es un animal de sangre fría parecida a sus primos marítimos y se mantiene así porque permanece completamente humedecida.

Nutrición.- Casi todas las especies de oligoquetos, tanto acuáticos como terrestres, se alimentan de materias orgánicas muertas, especialmente vegetales. La lombriz de tierra come materias descompuestas de la superficie, pero también utiliza sustancias orgánicas que obtiene en el lodo o cieno que ingiere al excavar.

Reproducción.- Los oligoquetos son hermafroditas, poseen gónadas separadas y el número de segmentos reproductores es sumamente limitado.

El plan general del sistema reproductor de los oligoquetos es relativamente uniforme, aunque son sumamente variables los números de las diversas estructuras, los segmentos en que se localizan y aquellos en que se abren los poros genitales, esta variación tiene importancia considerable en la taxonomía de los oligoquetos.

Clitelo.- Recibe este nombre una estructura reproductiva característica de los oligoquetos, incluye algunos segmentos vecinos en los cuales la epidermis aparece 
turgente por la presencia de glándulas unicelulares que forman un cinturón que rodea su porción dorsal hacia abajo.

En muchas lombrices terrestres constituyen un fuerte cinturón, el desarrollo del clitelo generalmente coincide con la madurez sexual, pero en algunos vermes sólo se manifiesta netamente durante la estación de la crianza.

Cópula.- En la oligoquetos la cópula es la regla con diseminación mutua de espermatozoos, durante la misma se ponen en contacto las superficies ventrales anteriores de un par de gusanos, con la parte anterior de uno de ellos dirigida hacia la posterior del otro; en todos los oligoquetos, salvo en los lumbricidos, los poros genitales masculinos de un yerme se yuxtaponen directamente a los receptáculos seminales del otro, siendo mantenidos ambos en posición por una envoltura mucosa común secretada por el clitelo.

El movimiento descendente de los espermotozoos por el surco espermático tiene lugar gracias a una mayor contracción de los músculos que producen el surco, se forma así una pequeña depresión que amplía la longitud del mismo llevando pequeñas cantidades de semen a los receptáculos seminales. La emisión de semen puede ser simultáneo o, por el contrario, no coincidir. En lumbricus, el fenómeno copulatorio se prolonga de dos a tres horas.

\section{MATERIALES Y METODOS}

\section{Ubicación del área experimental}

El experimento se ejecutó en la estación del IIAP-Ucayali, situado en el km 12400 de la carretera Pucallpa-Lima en la Región Ucayali. Las coordenadas son 8 22'31' de latitud sur y $74^{\circ} 34^{\prime} 35^{\prime}$ ' de longitud oeste. La altitud es de $154 \mathrm{msnm}$. El estudio se realizó de abril a setiembre de 1995.

Ecológicamente y de acuerdo con el estudio realizado por el INRENA, el experimento se encuentra en la formación ecológica denominada Bosque Húmedo Tropical. 


\section{Características meteorológicas del área}

Las características meteorológicas del área son:

Temperatura promedio mensual $\quad: 25,8^{\circ} \mathrm{C}$.

Precipitación promedio anual $\quad: 1324 \mathrm{~mm}$.

Humedad relativa promedio anual $: 87 \%$

(Fuente: Universidad de Ucayali).

\section{MATERIALES}

\section{Materiales experimentales}

Para la ejecución se contó con lombrices clasificadas como anélidos terrestres de la clase oligoquetos, siendo la especie Eísenia foetida y la lombriz nativa recolectadas de los terrenos de la Estación.

\section{Materiales}

Tapers plásticos.

Agua.

Guano de ganado vacuno.

Maleza (pasto torurco).

Materia orgánica del horizonte A1, A2.

\section{Instrumentos de control}

Termómetro.

Peachímetro.

Higrómetro.

Balanza.

Otros de laboratorio.

\section{Metodología del experimento}

El presente trabajo trató de demostrar que si unimos una lombriz de Eisenia foetida (que vive en cautiverio) con una lombriz nativa de mayor tamaño, se lograría el cruce mediante la metodología que exponemos a continuación. 


\section{Fases}

\section{Cuarentena}

Se seleccionó un total de 24 lombrices adultas, 12 nativas y 12 de Eisenia foetida pero teniendo en consideración similitud de caracteres fenotípicos, color, forma, etc., se les sometió a un aislamiento individual a fin de eliminar las cápsulas que habían sido fecundadas en su hábitat natural.

\section{Siembra de lombrices}

Consistió en lograr la cópula y postura de cápsulas de los siguientes tratamientos:

$\mathrm{T} 1=\operatorname{Ln} \times \operatorname{Ln}=2 \operatorname{Ln} \times 4$ repeticiones $=8 \mathrm{Ln}$

$\mathrm{T} 2=\mathrm{Ef} \times \mathrm{Ef}=2 \mathrm{Ef} \times 4$ repeticiones $=8 \mathrm{Ef}$

$\mathrm{T} 3=\mathrm{Ln} \times \mathrm{Ef}=1 \mathrm{Ln} \times 1 \mathrm{Ef} \times 4$ repeticiones $=4 \mathrm{Ln}$ y $4 \mathrm{Ef}$

\section{Incubación}

Se realiza cuando T3 presenta postura de cápsulas en un período de 21 días, luego el retiro de las lombrices madres que hayan logrado la postura de huevos. Esta fase abarca el período de incubación, es decir, hasta la eclosión de las cápsulas. La aparición de cápsulas en el T3 será indicativa de cruce; de no existir cápsulas hasta los 60 días se descarta la lombriz nativa.

\section{Crecimiento}

Se inicia después de la eclosión de las cápsulas hasta la madurez sexual, con aparición del clitelo.

\section{Reproducción de híbrido (F1)}

Abarca un nuevo proceso de reproducción que consiste en propagar una generación híbrida (F1), para ello se selecciona diez lombrices adultas producto del cruce inicial.

Se juntan de par en par en cinco repeticiones, se repite el proceso de la Fase 2 a la 3 . 


\section{Prueba final}

Con la progenie F2, lograda a partir de F1, se evaluó los siguientes tratamientos:

T1 $=2$ LN x 4 repeticiones.

$\mathrm{T} 2=2$ Ef $\mathrm{x} 4$ repeticiones.

$\mathrm{T} 3=2 \mathrm{~F} 2 \times 4$ repeticiones.

\section{Variables a medir}

Velocidad de humificación del alimento.

Cantidad lograda de cápsulas y lombrices por tratamiento.

Período de incubación.

Peso total de progenie adulta.

Medida de la progenie adulta.

Cantidad de alimento consumido por tratamiento.

\section{RESULTADOS}

Sólo se ejecutó hasta la segunda fase (siembra de lombrices) ya que no hubo cruce entre Ln y Ef (T3) que era el objetivo principal del estudio.

1. CUARENTENA: POSTURA DE CAPSULA DE LOMBRICES

\begin{tabular}{|l|c|c|c|c|c|c|c|c|c|c|c|c|c|c|}
\hline \multicolumn{1}{|c|}{ ESPECIES } & \multicolumn{10}{|c|}{} \\
\hline & 1 & 2 & 3 & 4 & 5 & 6 & 7 & 8 & 9 & 10 & 11 & 12 & Total & x \\
\hline Ef & 4 & 5 & 3 & 4 & 4 & 5 & 4 & 3 & 4 & 4 & 5 & 3 & 48 & 4,0 \\
Ln & 1 & 2 & 1 & 1 & 2 & 1 & 1 & 2 & 1 & 2 & 1 & 1 & 16 & 1,3 \\
\hline
\end{tabular}




\section{BIBLIOGRAFIA}

BARNES, ROBERT D. 1969. Zoología de los invertebrados. Segunda Edición. $737 \mathrm{pp}$.

BANCO AGRARIO DEL PERU. 1988. Manual de Instrucciones para la Lombricultura. Lima: Departamento de divulgación técnica. 25 p.p

FERRUZZ1, C. 1987. Manual de Lombricultura. Madrid: Mundo Prensa. 130 pp.

GUTIERREZ, J. 1977. Lombricultura; Alternativa ambientalista socio-económica ilimitada. Lima: CONCYTEC.

LA RECHERCHE. 1984. Los gusanos de tierra. En: Mundo Científico. Barcelona (España): Ed. Fontalba S.A.

BARNES, ROBERT D. 1969. Zoología de los invertebrados. Segunda edición. Editorial Interamericana S.A. 737 pp.

LAISSUS B., V. 1985. La Lombricultura. Paris (Francia): Montfermeil.

ZARELA, OLGA; RIOS, SONIA; SANCHEZ, MIGUEL. 1993. Manual de Lombricultura en Trópico Húmedo. Iquitos. 